\title{
Effect of metformin monotherapy on serum lipid profile in statin-naïve individuals with newly diagnosed type 2 diabetes mellitus: A cohort study
}

\author{
Szu Han Lin ${ }^{1}$, Po Chung Cheng ${ }^{1}$, Shih Te Tu ${ }^{1}$, Shang Ren Hsu ${ }^{\text {Corresp., }}{ }^{1}$, Yun Chung Cheng ${ }^{2}$, Yu Hsiu Liu \\ 1 Division of Endocrinology and Metabolism, Department of Internal Medicine, Changhua Christian Hospital, Changhua City, Taiwan \\ 2 Department of Radiology, Taichung Veterans General Hospital, Taichung, Taiwan \\ 3 Department of Accounting and Information Systems, National Taichung University of Science and Technology, Taichung, Taiwan \\ Corresponding Author: Shang Ren Hsu \\ Email address: 67781@cch.org.tw
}

Background. Cardiovascular disease is a major cause of mortality and morbidity in people with type 2 diabetes mellitus (T2DM). Studies have consistently identified dyslipidemia as an important risk factor for the development of macrovascular disease. The landmark United Kingdom Prospective Diabetes Study has shown that metformin therapy reduces cardiovascular events in overweight people with T2DM. This study investigates the effect of metformin monotherapy on serum lipid profile in statin-naïve individuals with newly diagnosed T2DM, and whether the effect, if any, is dosage-related. Methods. This cohort study enrolled individuals exceeding 20 years of age, with recent onset T2DM, who received at least 12 months of metformin monotherapy and blood tests for serum lipid at 6-month intervals. Exclusion criteria involved people receiving any additional antidiabetic medication or lipid-lowering drug therapy. Lipid-modifying effect of metformin was recorded as levels of serum triglycerides (TG), high density lipoprotein cholesterol (HDL-C), and low density lipoprotein cholesterol (LDL-C) measured at six month intervals. Results. The study enrolled 155 participants with a mean age of 58.6 years and average glycosylated hemoglobin $A_{1 c}$ of $8 \%$. After initiating metformin therapy, LDL-C was significantly reduced from $111 \mathrm{mg} / \mathrm{dl}$ to $102 \mathrm{mg} / \mathrm{dL}$ at 6 months $(P<0.001)$, TG was reduced from $132 \mathrm{mg} / \mathrm{dl}$ to $122 \mathrm{mg} / \mathrm{dL}$ at 12 months $(P=0.046)$, and HDL-C increased from $45.1 \mathrm{mg} / \mathrm{dL}$ to $46.9 \mathrm{mg} / \mathrm{dL}$ at 12 months $(P=0.02)$. However, increasing the dosage of metformin yielded no significant effect on its lipid-lowering efficacy. Discussion. Metformin monotherapy appreciably improves dyslipidemia in statin-naive people with T2DM. Its lipid-modifying effect may be attributable to insulin sensitization, reduction of irreversibly glycated LDL-C, and weight loss. In practice, people with dyslipidemia who are ineligible for lipid-lowering agents may benefit from metformin therapy. Moreover, previous studies report a synergistic effect between metformin and statin, which may 
further reduce cardiovascular events in at-risk individuals. Overall, metformin is a safe and efficacious approach to alleviate dyslipidemia in people with newly diagnosed T2DM. 
1

2

3 Effect of metformin monotherapy on serum lipid profile in statin-naïve individuals with newly

4

5

6 Szu Han Lin ${ }^{1}$, Po Chung Cheng ${ }^{1}$, Shih Te Tu${ }^{1}$, Shang Ren Hsu ${ }^{1}$, Yun Chung Cheng ${ }^{2}$, Yu Hsiu 7

8

$9 \quad{ }^{1}$ Division of Endocrinology and Metabolism, Department of Internal Medicine, Changhua

10 Christian Hospital, 135 Nanxiao St., Changhua City, Changhua County, Taiwan

$11{ }^{2}$ Department of Radiology, Taichung Veterans General Hospital, 1650 Taiwan Boulevard

12 Section 4, Taichung, Taiwan

13

14

15

16

17 Email address: 67781@cch.org.tw

18

19

20

21

22

23

24

Author Cover Page

$\mathrm{Liu}^{3}$

${ }^{3}$ Department of Accounting and Information Systems, National Taichung University of Science and Technology, 129 San Min Rd., Taichung, Taiwan

Corresponding author: Shang Ren Hsu 
26

27

28

29

30

31

32

33

34

35

36

37

38

39

40

41

42

43

44

Abstract

Background. Cardiovascular disease is a major cause of mortality and morbidity in people with type 2 diabetes mellitus (T2DM). Studies have consistently identified dyslipidemia as an important risk factor for the development of macrovascular disease. The landmark United Kingdom Prospective Diabetes Study has shown that metformin therapy reduces cardiovascular events in overweight people with T2DM. This study investigates the effect of metformin monotherapy on serum lipid profile in statin-naïve individuals with newly diagnosed T2DM, and whether the effect, if any, is dosage-related.

Methods. This cohort study enrolled individuals exceeding 20 years of age, with recent onset T2DM, who received at least 12 months of metformin monotherapy and blood tests for serum lipid at 6-month intervals. Exclusion criteria involved people receiving any additional antidiabetic medication or lipid-lowering drug therapy. Lipid-modifying effect of metformin was recorded as levels of serum triglycerides (TG), high density lipoprotein cholesterol (HDL-C), and low density lipoprotein cholesterol (LDL-C) measured at six month intervals.

Results. The study enrolled 155 participants with a mean age of 58.6 years and average glycosylated hemoglobin $\mathrm{A}_{1 \mathrm{c}}$ of $8 \%$. After initiating metformin therapy, LDL-C was significantly reduced from $111 \mathrm{mg} / \mathrm{dl}$ to $102 \mathrm{mg} / \mathrm{dL}$ at 6 months $(P<0.001)$, TG was reduced from $132 \mathrm{mg} / \mathrm{dl}$ to $122 \mathrm{mg} / \mathrm{dL}$ at 12 months $(P=0.046)$, and HDL-C increased from $45.1 \mathrm{mg} / \mathrm{dL}$ to $46.9 \mathrm{mg} / \mathrm{dL}$ at 12 months $(P=0.02)$. However, increasing the dosage of metformin yielded no significant effect on its lipid-lowering efficacy.

7 Discussion. Metformin monotherapy appreciably improves dyslipidemia in statin-naive people with T2DM. Its lipid-modifying effect may be attributable to insulin sensitization, reduction of 
49 irreversibly glycated LDL-C, and weight loss. In practice, people with dyslipidemia who are 50 ineligible for lipid-lowering agents may benefit from metformin therapy. Moreover, previous

51 studies report a synergistic effect between metformin and statin, which may further reduce

52 cardiovascular events in at-risk individuals. Overall, metformin is a safe and efficacious

53 approach to alleviate dyslipidemia in people with newly diagnosed T2DM.

54

55 Keywords: type 2 diabetes mellitus, dyslipidemia, metformin

56

57

58

59

60

61

62

63

64

65

66

67

68

69

70

71

72 
73

74 Effect of metformin monotherapy on serum lipid profile in statin-naïve individuals with newly 75 diagnosed type 2 diabetes mellitus: A cohort study

76

77 Introduction

78

79 Cardiovascular disease causes substantial morbidity in people with type 2 diabetes mellitus 80 (T2DM) (Kannel \& McGee, 1979). Coronary heart disease not only leads to an increased 81 mortality rate (Martín-Timón et al., 2014) but also contributes to long-term cardiac dysfunction 82 (Miki et al., 2013). Studies have consistently identified dyslipidemia as an important risk factor 83 for the development of macrovascular disease in T2DM (Schofield et al., 2016; Stern, 1995; Wu $84 \&$ Parhofer, 2014). A safe and efficacious intervention for diabetic dyslipidemia is necessary to 85 attenuate cardiovascular disease in at-risk individuals.

86

87 Metformin is the first-line treatment for people with newly diagnosed T2DM endorsed by the 88 American Diabetes Association (American Diabetes Association, 2017). This medication 89 primarily inhibits hepatic gluconeogenesis, thereby reducing fasting glucose levels (Song, 2016).

90 Moreover, metformin is an antioxidant that diminishes cancer risk (Kasznicki, Sliwinska \&

91 Drzewoski, 2014) and improves insulin sensitivity (Shaw, 2013). Importantly, the landmark

92 United Kingdom Prospective Diabetes Study (UKPDS) has shown that metformin therapy 93 reduces cardiovascular events in overweight people with T2DM (American Diabetes Association, 94 2002).

95

96 Clinical trials have given hint to the lipid-modifying effect of metformin. In people with recent 
97 onset T2DM, metformin enhances the lipid-lowering efficacy of atorvastatin (Kashi et al., 2016).

98 A recent metabolomics study demonstrates that metformin may reduce low density lipoprotein

99 cholesterol (LDL-C) via an AMP-activated protein kinase pathway (Xu et al., 2015). However,

100 none of the published studies specifically excludes individuals receiving statin therapy or

101 second-line antidiabetic medications in addition to metformin. Considering the predominant

102 lipid-lowering effect of statin in people with T2DM (Colhoun et al., 2004), potential lipid-

103 modifying capacity of metformin is difficult to isolate from that of concomitant statin therapy.

104

105 This study investigates the effect of metformin monotherapy on serum lipid profile in statin-

106 naïve individuals with newly diagnosed T2DM. In addition, the study determines whether

107 metformin's lipid-modifying effect, if any, is dosage-related in these participants.

108

109 Materials and methods

110

111 This cohort study was conducted at Changhua Christian Hospital in central Taiwan. Participants

112 who visited the Endocrinology clinic between December 2013 and November 2015 were

113 screened for eligibility. Inclusion criteria were individuals exceeding 20 years of age, with recent

114 onset T2DM, who received at least 12 months of metformin monotherapy and blood tests for

115 serum lipid at 6-month intervals. Exclusion criteria involved people receiving second-line

116 antidiabetic medications or lipid-lowering drugs such as statin, fibrate, bile acid sequestrant,

117 nicotinic acid, or ezetimibe. Individuals with familial hypercholesterolemia, thyroid disorder,

118 renal dysfunction, or alcoholism were also ineligible. The study was approved by the

119 Institutional Review Board of Changhua Christian Hospital (CCH IRB: 180102). All participants

120 provided written consent to take part in the study. 
122 Demographic information including age, sex, and systolic blood pressure were recorded at 123 diagnosis of T2DM. Thereafter participants received blood tests for serum lipid at 6-month

124 intervals. Metformin dosage for each individual was defined as the daily quantity received for

125 the longest duration in the first year of treatment. Lipid-modifying effect of metformin was

126 recorded as levels of serum triglycerides (TG), high density lipoprotein cholesterol (HDL-C),

127 and low density lipoprotein cholesterol (LDL-C) measured at six month intervals. The lipid tests

128 in this study were performed using Beckman Coulter UniCel DxC 800 Synchron $^{\mathrm{TM}}$ Clinical

129 Systems. The analytical precision was within $1.7 \mathrm{mg} / \mathrm{dL}$ for HDL-C, within $3.0 \mathrm{mg} / \mathrm{dL}$ for LDL$130 \mathrm{C}$, and within $7.5 \mathrm{mg} / \mathrm{dL}$ for TG.

131

132 Paired $t$-test enabled comparison of participants' serum lipid profile at diagnosis and after 133 receiving metformin therapy. Levels of serum lipid were compared between dosage-based 134 subgroups using one-way analysis of variance. A two-tailed $P$ value of less than 0.05 indicated 135 statistical significance. Statistical analysis was performed using IBM SPSS version 22.0 (IBM 136 SPSS Statistics for Windows. Armonk, NY).

138 Results

140 The study screened 180 individuals for eligibility. Eighteen patients were excluded due to

141 prescription of second-line antidiabetic medications, and seven were ineligible because they

142 received lipid-lowering drugs. The enrollment process is illustrated in Figure 1.

144 The study enrolled 155 participants whose demographic features are summarized in Table 1. 
145 They had a mean age of 58.6 years and an average glycosylated hemoglobin $\mathrm{A}_{1 \mathrm{c}}\left(\mathrm{HbA}_{1 \mathrm{c}}\right)$ of $8 \%$.

146 In terms of serum lipid profile, participants harbored a mean LDL-C level of $111 \mathrm{mg} / \mathrm{dL}$, mean

147 HDL-C of $45.1 \mathrm{mg} / \mathrm{dL}$, and mean TG of $132 \mathrm{mg} / \mathrm{dL}$.

148

149 After initiating metformin therapy, LDL-C was significantly reduced from $111 \mathrm{mg} / \mathrm{dl}$ to 102

$150 \mathrm{mg} / \mathrm{dL}$ at 6 months $(P<0.001)$, TG was reduced from $132 \mathrm{mg} / \mathrm{dl}$ to $122 \mathrm{mg} / \mathrm{dL}$ at $12 \mathrm{months}(P$

$151=0.046)$, and HDL-C increased from $45.1 \mathrm{mg} / \mathrm{dL}$ to $46.9 \mathrm{mg} / \mathrm{dL}$ at $12 \mathrm{months}(P=0.02)$. After

15212 months of treatment, mean $\mathrm{HbA}_{1 \mathrm{c}}$ was reduced from $8 \%$ to $6.47 \%(P<0.001)$, and mean

153 body weight changed from 69 kilograms to 68.3 kilograms $(P=0.01)$. These findings are

154 tabulated in Table 2.

155

156 As shown in Table 3, increasing the dosage of metformin had no significant effect on its lipid-

157 lowering efficacy. Specifically, improvement in TG, HDL-C, and LDL-C was similar between

158 participants receiving $1000 \mathrm{mg}, 1500 \mathrm{mg}$, and $2000 \mathrm{mg}$ of metformin per day. At the end of this

159 study, all three subgroups had comparable mean $\mathrm{HbA}_{1 \mathrm{c}}$, while those receiving $2000 \mathrm{mg}$ of

160 metformin per day harbored higher mean body weight.

161

162 Discussion

163

164 Metformin may counter the derangements in lipid metabolism in T2DM through several

165 pathways (Malin et al., 2012; Han \& Kaufman, 2016). Through increasing insulin sensitivity,

166 metformin reduces the rate of lipolysis, thereby slowing the conversion of free fatty acids to

167 lipoprotein precursors in the liver (Melmed et al., 2016). By reducing plasma glucose levels,

168 metformin lowers the fraction of irreversibly glycated LDL-C, which is removed less efficiently 
169 from the body (Sima et al., 2010). Metformin also improves dyslipidemia by inducing weight

170 loss in people with impaired glucose metabolism (Diabetes Prevention Program Research Group,

171 2012; Harder, Dinesen \& Astrup, 2004). Following metformin treatment, weight loss is in

172 general modest and attributable to fat loss rather than to energy expenditure (Yanovski et al,

173 2011).

174

175 As observed in the current study, metformin monotherapy lowered LDL-C level after only 6

176 months of therapy, whereas serum TG and HDL-C did not significantly improve until after 12

177 months. This lipid-modifying effect is concordant with existing evidence that

178 hypertriglyceridemia and diminished HDL-C require a longer therapeutic duration to counteract

179 than lowering LDL-C (Orchard et al., 2005). Moreover, metformin's effect on serum lipid

180 appeared to be dosage-independent in this study, suggesting this medication may control

181 dyslipidemia through indirect pathways.

182

183 Clinical implications arise from the observation that metformin monotherapy improves

184 dyslipidemia in diabetic patients. Metformin not only controls hyperglycemia but also reduces

185 cardiovascular risk, as shown previously in the UKPDS (American Diabetes Association, 2002).

186 Considering the cost and potential side effects of lipid-lowering medications such as statins

187 (Raymond et al., 2014), people with dyslipidemia who are ineligible for lipid-lowering therapy

188 may nonetheless benefit from metformin treatment. Previous investigators have also observed a

189 synergistic effect between metformin and statin (Kashi et al., 2016), which can further reduce

190 cardiovascular events in at-risk individuals.

191

192 As demonstrated in this study, metformin modifies serum lipid in a dosage-independent manner. 
193 Considering that the gastrointestinal side effect of metformin occurs more frequently at higher

194 dosage (McCreight, Bailey \& Pearson, 2016), people who experience such side effect may

195 benefit from a lower dosage of metformin, which nonetheless provides lipid-lowering effect.

196 Moreover, in people with adequate glycemic control using second-line antidiabetic medications,

197 adding metformin at low dose may further reduce their cardiovascular risk through the beneficial 198 effect on lipid profile.

200 This study differs from previous investigations of metformin therapy that did not specifically 201 exclude recipients of lipid-lowering medications, which obviously constitute a confounder on 202 serum lipid profile. The current study reduces potential confounders by excluding recipients of 203 either lipid-lowering drugs or second-line antidiabetic agents. Moreover, the participants have 204 received follow up at the same medical center throughout the study, which circumvents 205 variations in laboratory test results due to different analytic methods.

207 Several limitations arise from the study design. Therapeutic lifestyle change is an essential 208 component of dyslipidemia treatment (Mannu et al., 2013). Since all participants received self209 management education by diabetes educators, a beneficial effect of metformin on serum lipid is 210 difficult to distinguish from that of lifestyle modification. Indeed, the lack of a control group 211 receiving only lifestyle intervention constitutes an important limitation in this study. However, 212 considering that early treatment to glycemic target substantially improves clinical outcome 213 (American Diabetes Association, 2017), establishing a control group without any antidiabetic 214 medication may be inappropriate at diagnosis of T2DM.

216 Another limitation is that serum lipid levels may change as a consequence of anti-hyperglycemic 
217 drugs (Buse et al., 2004), exercise (Balducci et al., 2009), and diet (Greco M et al., 2014).

218 However, the influence of exercise and diet on serum lipid levels was not addressed by the study.

219 Finally, the mechanism behind metformin's lipid-modifying effect remains elusive, and further 220 studies are required to explore the associated metabolic pathways.

221

222 Conclusions

223

224 In people with newly diagnosed T2DM, metformin therapy significantly reduced both serum

225 LDL-C and TG, as well as raised HDL-C, without concomitant lipid-lowering medications.

226 Moreover, the lipid-modifying effect of metformin appeared to be dosage-independent. Overall,

227 metformin is a safe and efficacious approach to alleviate dyslipidemia in people with T2DM.

228

229 Acknowledgement

230

231 None.

232

233 References

234

235

American Diabetes Association. 2017. Standards of medical care in diabetes—2017: summary

236 of revisions. Diabetes Care. 40(Supplement 1): S4-S5.

237 American Diabetes Association. 2002. Implications of the United Kingdom Prospective

238 Diabetes Study. Diabetes Care. 25(suppl 1): s28-s32.

239 Balducci S, Zanuso S, Fernando F, Fallucca S, Fallucca F, Pugliese G. 2009. Physical

240 activity/exercise training in type 2 diabetes. The role of the Italian Diabetes and Exercise Study. 
241 Diabetes Metab Res Rev. 25(Suppl 1):S29-33.

242 Buse JB, Tan MH, Prince MJ, Erickson PP. 2004. The effects of oral anti-hyperglycaemic

243 medications on serum lipid profiles in patients with type 2 diabetes. Diabetes Obes Metab.

$244 \quad 6: 133-156$.

245 Colhoun HM1, Betteridge DJ, Durrington PN, Hitman GA, Neil HA, Livingstone SJ,

246 Thomason MJ, Mackness MI, Charlton-Menys V, Fuller JH. 2004. Primary prevention of

247 cardiovascular disease with atorvastatin in type 2 diabetes in the Collaborative Atorvastatin

248 Diabetes Study (CARDS): multicentre randomised placebo-controlled trial. Lancet. 364:685-696.

249 Diabetes Prevention Program Research Group. 2012. Long-term safety, tolerability, and

250 weight loss associated with metformin in the Diabetes Prevention Program Outcomes Study.

251 Diabetes Care. 35:731-737.

252 Greco M, Chiefari E, Montalcini T, Accattato F, Costanzo FS, Pujia A, Foti D, Brunetti A,

253 Gulletta E. 2014. Early effects of a hypocaloric, Mediterranean diet on laboratory parameters in

254 obese individuals. Mediators Inflamm. Article ID 2014:750860.

255 Han J, Kaufman RJ. 2016. The role of ER stress in lipid metabolism and lipotoxicity. J Lipid 256 Res. 57:1329-1338.

257 Harder H, Dinesen B, Astrup A. 2004. The effect of a rapid weight loss on lipid profile and 258 glycemic control in obese type 2 diabetic patients. Int J Obes Relat Metab Disord. 28:180-182.

259 Kannel WB, McGee DL. 1979. Diabetes and cardiovascular disease. The Framingham study. 260 JAMA. 241:2035-2038.

261 Kashi Z, Mahrooz A, Kianmehr A, Alizadeh A. 2016. The role of metformin response in lipid

262 metabolism in patients with recent-onset type 2 diabetes: HbAlc level as a criterion for

263 designating patients as responders or nonresponders to metformin. PLoS One. 11:e0151543.

264 Kasznicki J, Sliwinska A, Drzewoski J. 2014. Metformin in cancer prevention and therapy. 
265 Annals of Translational Medicine. 2:57-68.

266 Malin SK, Gerber R, Chipkin SR, Braun B. 2012. Independent and combined effects of

267 exercise training and metformin on insulin sensitivity in individuals with prediabetes. Diabetes

268 Care. 35:131-136.

269 Mannu GS, Zaman MJ, Gupta A, Rehman HU, Myint PK. 2013. Evidence of lifestyle

270 modification in the management of hypercholesterolemia. Curr Cardiol Rev. 9:2-14.

271 Martín-Timón I, Sevillano-Collantes C, Segura-Galindo A, del Cañizo-Gómez FJ. 2014.

272 Type 2 diabetes and cardiovascular disease: Have all risk factors the same strength? World

273 Journal of Diabetes. 5:444-470.

274 McCreight LJ, Bailey CJ, Pearson ER. 2016. Metformin and the gastrointestinal tract.

275 Diabetologia. 59:426-435.

276 Melmed S, Polonsky KS, Larsen PR, Kronenberg HM. 2016. Williams Textbook of

277 Endocrinology, 13th Edition. Chapter 37: Disorders of lipid metabolism (Page 1662-1665).

278 Elsevier.

279 Miki T, Yuda S, Kouzu H, Miura T. 2013. Diabetic cardiomyopathy: pathophysiology and 280 clinical features. Heart Fail Rev. 18:149-66.

281 Orchard TJ, Temprosa M, Goldberg R, Haffner S, Ratner R, Marcovina S, Fowler S. 2005.

282 The effect of metformin and intensive lifestyle intervention on the metabolic syndrome: The

283 Diabetes Prevention Program randomized trial. Annals of Internal Medicine. 142:611-619.

284 Raymond C, Cho L, Rocco M, Hazen SL. 2014. New guidelines for reduction of blood

285 cholesterol: Was it worth the wait? Cleveland Clinic Journal of Medicine. 81: 11-19.

286 Schofield JD, Liu Y, Rao-Balakrishna P, Malik RA, Soran H. 2016. Diabetes dyslipidemia.

287 Diabetes Ther. 7: 203-219.

288 Shaw RJ. 2013. Metformin trims fats to restore insulin sensitivity. Nat Med. 19: 1649-1654. 
289 Sima AV, Botez GM, Stancu CS, Manea A, Raicu M, Simionescu M. 2010. Effect of

290 irreversibly glycated LDL in human vascular smooth muscle cells: lipid loading, oxidative and

291 inflammatory stress. Journal of Cellular and Molecular Medicine. 14:2790-2802.

292 Song R. 2016. Mechanism of metformin: A tale of two sites. Diabetes Care. 39: 187-189.

293 Stern MP. 1995. Diabetes and cardiovascular disease. The "common soil" hypothesis. Diabetes.

294 44:369-374.

295 Wu L, Parhofer KG. 2014. Diabetic dyslipidemia. Metabolism. 63:1469-1479.

296 Xu T, Brandmaier S, Messias AC, Herder C, Draisma HH, Demirkan A, Yu Z, Ried JS,

297 Haller T, Heier M, Campillos M, Fobo G, Stark R, Holzapfel C, Adam J, Chi S, Rotter M,

298 Panni T, Quante AS, He Y, Prehn C, Roemisch-Margl W, Kastenmüller G, Willemsen G,

299 Pool R, Kasa K, van Dijk KW, Hankemeier T, Meisinger C, Thorand B, Ruepp A, Hrabé

300 de Angelis M, Li Y, Wichmann HE, Stratmann B, Strauch K, Metspalu A, Gieger C, Suhre

301 K, Adamski J, Illig T, Rathmann W, Roden M, Peters A, van Duijn CM, Boomsma DI,

302 Meitinger T, Wang-Sattler R. 2015. Effects of metformin on metabolite profiles and LDL

303 cholesterol in patients with type 2 diabetes. Diabetes Care. 38:1858-1867.

304 Yanovski JA, Krakoff J, Salaita CG, McDuffie JR, Kozlosky M, Sebring NG, Reynolds JC,

305 Brady SM, Calis KA. 2011. Effects of metformin on body weight and body composition in

306 obese insulin-resistant children: A randomized clinical trial. Diabetes. 60: 477-485. 
Figure 1

Enrollment protocol for the study

Number of participants enrolled in the study and reason for exclusion

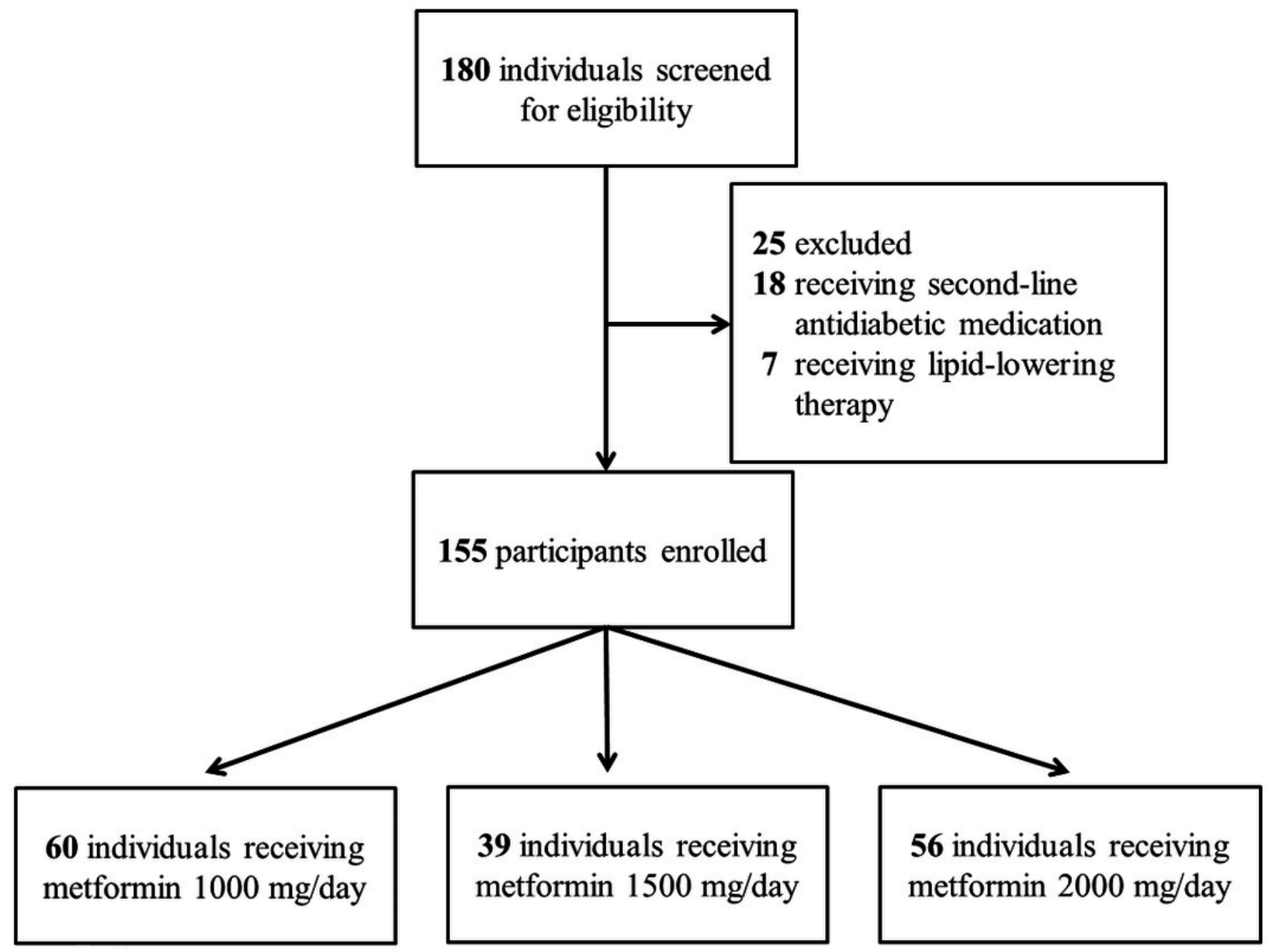




\section{Table $\mathbf{1}$ (on next page)}

Demographic features of participants at diagnosis of type 2 diabetes mellitus

Data are expressed as mean with standard deviation for continuous variables and number

(\%) for categorical variables. ALT: alanine aminotransferase, $\mathrm{HbA}_{1 c}$ : glycosylated hemoglobin $A_{1 c}$ 
1 Table 1. Demographic features of participants at diagnosis of type 2 diabetes mellitus 2

\begin{tabular}{ll}
\hline Variables & Study population $(n=155)$ \\
\hline
\end{tabular}

Age (years)

$$
58.6 \pm 13.4
$$

Sex (Female)

$77(49.7 \%)$

$\mathrm{HbA}_{1 \mathrm{c}}(\%)$

$$
8.0 \pm 1.8
$$

Creatinine $(\mathrm{mg} / \mathrm{dL})$

$$
0.84 \pm 0.22
$$

$\operatorname{ALT}(\mathrm{U} / \mathrm{mL})$

Systolic blood pressure

(mm Hg)

Metformin dose

(mg per day)

Microalbuminuria

(mg per day)

Triglycerides (mg/dL)

High density lipoprotein

cholesterol (mg/dL)

Low density lipoprotein cholesterol (mg/dL)
$38.4 \pm 27.0$

$133 \pm 16.3$

$1487 \pm 433.8$

$51.1 \pm 149$

$132 \pm 71.9$

$45.1 \pm 12.0$

$111 \pm 32.3$

3 Data are expressed as mean with standard deviation for continuous variables and number (\%) for

4 categorical variables. ALT: alanine aminotransferase, $\mathrm{HbA}_{1 \mathrm{c}}$ : glycosylated hemoglobin $\mathrm{A}_{1 \mathrm{c}}$ 5 


\section{Table 2 (on next page)}

Lipid-modifying effect of metformin in people with newly diagnosed type 2 diabetes mellitus

Data are expressed as mean with standard deviation for continuous variables. Variables are compared to baseline levels using paired $t$-test. 
1 Table 2. Clinical effect of metformin in people with newly diagnosed type 2 diabetes mellitus

\begin{tabular}{lcc}
$\begin{array}{l}\text { Treatment duration } \\
(\text { months })\end{array}$ & $\begin{array}{c}\text { Study population } \\
(n=155)\end{array}$ & $P$ value \\
\hline $\begin{array}{l}\text { Triglycerides }(\mathrm{mg} / \mathrm{dL}) \\
0\end{array}$ & $132 \pm 71.9$ & \\
6 & $123 \pm 66.2$ & 0.055 \\
12 & $122 \pm 63.6$ & 0.046
\end{tabular}

\section{High density lipoprotein}

cholesterol (mg/dL)

0

$45.1 \pm 12.0$

6

$46.3 \pm 13.3 \quad 0.076$

12

$46.9 \pm 13.7 \quad 0.020$

Low density lipoprotein

cholesterol (mg/dL)

0

$111 \pm 32.3$

6

$102 \pm 25.4$

0.001

12

$102 \pm 25.0$

0.001

\section{Glycated hemoglobin}

Alc (\%)

0

$8.00 \pm 1.83$

6

$6.62 \pm 0.88$

0.001

12

$6.47 \pm 0.68$

0.001

Body weight (kilograms)

0

$69.0 \pm 14.5$

12

$68.3 \pm 14.4$

0.010 
2 Data are expressed as mean with standard deviation for continuous variables. Variables are 3 compared to baseline levels using paired $t$-test. 


\section{Table 3(on next page)}

Comparison of lipid-modifying effect of metformin between dose-based subgroups

Data are expressed as mean with standard deviation for continuous variables. Variables are compared between dose-based subgroups using analysis of variance. 
1 Table 3. Comparison of clinical effect of metformin between dose-based subgroups

\begin{tabular}{lcccc}
\hline $\begin{array}{l}\text { Treatment duration } \\
\text { (months) }\end{array}$ & $\begin{array}{c}\text { Metformin } \\
1000 \mathrm{mg} / \mathrm{day} \\
(n=60)\end{array}$ & $\begin{array}{c}\text { Metformin } \\
1500 \mathrm{mg} / \mathrm{day} \\
(n=39)\end{array}$ & $\begin{array}{c}\text { Metformin } \\
2000 \mathrm{mg} / \mathrm{day} \\
(n=56)\end{array}$ & $P$ value \\
\hline $\begin{array}{l}\text { Triglycerides (mg/dL) } \\
0\end{array}$ & $131 \pm 60.3$ & $138 \pm 86$ & $130 \pm 71.9$ & 0.854 \\
6 & $121 \pm 71.6$ & $128 \pm 62.8$ & $121 \pm 63.5$ & 0.841 \\
12 & $118 \pm 58.7$ & $129 \pm 68.7$ & $120 \pm 65.7$ & 0.717 \\
\hline $\begin{array}{l}\text { High density lipoprotein } \\
\text { cholesterol (mg/dL) }\end{array}$ & & & & \\
0 & $44.9 \pm 13.3$ & $46.3 \pm 10.6$ & $44.5 \pm 11.5$ & 0.748 \\
6 & $47.0 \pm 15.0$ & $47.5 \pm 13.9$ & $44.6 \pm 10.9$ & 0.510 \\
12 & $47.0 \pm 14.1$ & $47.5 \pm 15.5$ & $46.3 \pm 11.8$ & 0.910 \\
\hline $\begin{array}{l}\text { Low density lipoprotein } \\
\text { cholesterol (mg/dL) }\end{array}$ & & & & \\
0 & $112 \pm 39.2$ & $107 \pm 28.4$ & $111 \pm 26.4$ & 0.725 \\
6 & $101 \pm 24.8$ & $101 \pm 27.5$ & $105 \pm 24.7$ & 0.691 \\
12 & $102 \pm 26.8$ & $100 \pm 21.3$ & $103 \pm 25.6$ & 0.859 \\
\hline & & & & \\
\hline
\end{tabular}

Glycated hemoglobin

A1c (\%)

0

$7.76 \pm 1.81 \quad 7.90 \pm 1.31$

$8.30 \pm 2.13 \quad 0.276$

6

$6.78 \pm 0.93$

$6.49 \pm 0.79$

$6.53 \pm 0.89$

0.180

12

$6.55 \pm 0.69$

$6.42 \pm 0.67$

$6.42 \pm 0.67$

0.527

Body weight (kilograms)

0

$64.8 \pm 13.2 \quad 67.4 \pm 12.8 \quad 74.8 \pm 15.4 \quad 0.001$ 


$$
64.2 \pm 13.9 \quad 66.4 \pm 12.1 \quad 74.0 \pm 15.0 \quad 0.001
$$

2 Data are expressed as mean with standard deviation for continuous variables. Variables are 3 compared between dose-based subgroups using analysis of variance. 